\title{
FINITE SUBGROUPS OF FORMAL $A$-MODULES OVER P-ADIC INTEGER RINGS
}

\author{
BY \\ TETSUO NAKAMURA
}

\begin{abstract}
Let $B \supset A$ be $\mathfrak{p}$-adic integer rings such that $A / Z_{p}$ is finite and $B / A$ is unramified. Generalizing a result of Fontaine on finite commutative p-group schemes, we show that galois homomorphisms of finite subgroups of one-dimensional formal $A$-modules over $B$ are given by power series.
\end{abstract}

Introduction. Let $K$ be a finite extension of the rational $p$-adic number field $Q_{p}$, and $A$ the integer ring of $K$. Let $L$ be a complete unramified extension of $K$, $B$ the ring of integers of $L$, and $\mathfrak{p}$ the maximal ideal of $B$. We write $\overline{\mathfrak{p}}$ for the maximal ideal of the integer ring of the algebraic closure $\bar{L}$ of $L$. Let $F$ denote an $n$-dimensional formal $A$-module defined over $B$ of finite $A$-height. Then $F$ induces an $A$-module structure on $\overline{\mathfrak{p}}^{n}$, which we denote by $F(\overline{\mathfrak{p}})$; it is an $A[\mathfrak{G}]$-module, where $\mathfrak{G}=\operatorname{Gal}(\bar{L} / L)$. Let $P$ be a finite sub- $A[\mathfrak{G}]$-module of $F(\overline{\mathfrak{p}})$ (henceforth, simply of $F$ ). In this paper, we attach to $P$ a couple $M L(P)$ of modules over a noncommutative power series ring. Let $G$ be another formal $A$-module over $B$ of finite $A$-height and $Q$ be a finite sub- $A[\mathfrak{G}]$-module of $G$. Then we describe the $A[\mathfrak{G}]$ homomorphisms from $P$ to $Q$ by morphisms from $M L(Q)$ to $M L(P)$ (Theorem 1). If $A=Z_{p}$ (the $p$-adic integer ring), this result follows from Fontaine [4], but our proof depends rather on Tate modules of formal groups. Furthermore, if $F$ and $G$ are one-dimensional, we can show that every $A[\mathfrak{G}]$-homomorphism from $P$ to $Q$ is of the form $g^{-1} \circ c f$ for some $c \in B$, where $f$ and $g$ are the logarithms of $F$ and $G$, respectively (Theorem 3 ). In [8], Lubin has obtained a rather weaker version of this result.

In the following, let $K, A, L, B, \mathfrak{p}, \overline{\mathfrak{p}}$ and $\mathfrak{G}$ be as above. We write $\pi$ for a fixed prime element of $A$ and $q$ for the cadinality of the residue field of $A$. Let $\sigma$ denote the Frobenius automorphism of $L / K$. We write $E=B_{\sigma}[[T]]$ for the ring of noncommutative power series ring over $B$ in a variable $T$ with respect to the multiplication rule: $T b=b^{\sigma} T$ for all $b \in B$. Call $F^{A}(B)$ the category of finite-dimensional formal $A$-modules over $B$ of finite $A$-height.

I would like to thank the referee for calling my attention to Lubin [8].

1. Homomorphisms of finite subgroups of formal $A$-modules. We write $T(F)$ for the Tate module of a formal $A$-module $F . T(F)$ is an $A[\mathfrak{G}]$-module, $A$ free of rank $h(=A$-height of $F)$. Let $D H^{\prime}$ be the category defined in Decauwert [2]. Let $M(F)$ and $L(F)$ be as in [2]; $M(F)$ is an $E$-module, $B$-free of rank $h$ and

Received by the editors February 15, 1983.

1980 Mathematics Subject Classification. Primary 14L05.

Key words and phrases. Formal module (group), Tate module, special element, logarithm of formal group. 
$L(F)$ is a sub- $B$-module of $M(F)$. The functor $M L(F)=(M(F), L(F))$ induces an antiequivalence between $F^{A}(B)$ and $D H^{\prime}[2$, Théorème 2].

Let $\alpha: F \rightarrow G$ be a morphism in $F^{A}(B)$. We also write $\alpha$ for the homomorphism $T(F) \rightarrow T(G)$ induced by $\alpha$. We write $\tilde{\alpha}$ for the morphism $M L(G) \rightarrow M L(F)$ induced by $\alpha$.

LEMMA 1. Let $F, G$ and $H$ be objects of $F^{A}(B)$. Let $\alpha: F \rightarrow H$ and $\beta: H \rightarrow G$ be homomorphisms over $B$. Then $0 \rightarrow T(F) \stackrel{\alpha}{\rightarrow} T(H) \stackrel{\beta}{\rightarrow} T(G) \rightarrow 0$ is exact if and only if $0 \rightarrow M L(G) \stackrel{\tilde{\beta}}{\rightarrow} M L(H) \stackrel{\tilde{\alpha}}{\rightarrow} M L(F) \rightarrow 0$ is exact.

SKETCH OF PROOF. For a morphism $s$ in $D H^{\prime}$, we see that Ker $s$ and $\operatorname{Im} s$ are in $D H^{\prime}$. The "if" part follows easily from this. By Fontaine [5, Chapter V, §2] we can express $M L(F)$ by means of special elements. Choosing an appropriate special element of $H$, we can prove the "only if" part (cf. also Honda [6]).

Now let $F \in F^{A}(B)$, and let $P$ be a finite sub- $A[\mathfrak{G}]$-module of $F$. Denote by $S$ the superlattice of $T(F)$ in $T(F) \otimes_{A} K$ such that $S / T(F) \cong P$. Then by Waterhouse [10, Theorem 1.3] there exists an isogeny $\nu: F \rightarrow F^{\prime}$ defined over $B$ such that $S=\nu^{-1} T\left(F^{\prime}\right)$. As $S$ is an $A[\mathfrak{G}]$-module, we see that $F^{\prime} \in F^{A}(B)$. Define $M L(P)=$ $(M(P), L(P))$, where $M(P)=M(F) / \tilde{\nu} M\left(F^{\prime}\right)$ and $L(P)=L(F) / \tilde{\nu} L\left(F^{\prime}\right)$. Then $M(P)$ is an $E$-module and $L(P)$ is a sub-B-module of $M(P)$. Let $M, M^{\prime}$ be left $E$-modules and $N, N^{\prime}$ be sub- $B$-modules of $M$ and $M^{\prime}$, respectively. By $\operatorname{Hom}_{E}\left((M, N),\left(M^{\prime}, N^{\prime}\right)\right)$ we denote the set of $E$-linear maps $\delta: M \rightarrow M^{\prime}$ such that $\delta(N) \subset N^{\prime}$. Then clearly $P$ determines $M L(P)$ up to an $E$-isomorphism.

THEOREM 1. Let $F, G \in F^{A}(B)$. Let $P$ and $Q$ be finite sub-A[G]-modules of $F$ and $G$, respectively. Then $\operatorname{Hom}_{A[\mathfrak{b}\}}(P, Q)$ is $A$-isomorphic to

$$
\operatorname{Hom}_{E}(M L(Q), M L(P)) \text {. }
$$

Sketch of Proof. We refer to the method used in Oort [9]. Let $\alpha: F \rightarrow F^{\prime}$ and $\beta: G \rightarrow G^{\prime}$ be isogenies over $B$ such that $\operatorname{Ker} \alpha=P$ and $\operatorname{ker} \beta=Q$. Write $T_{1}=T(F), T_{2}=T(G), M_{1}=M L(F)$ and $M_{2}=M L(G)$; let $T_{1}^{\prime}, T_{2}^{\prime}, M_{1}^{\prime}, M_{2}^{\prime}$ be similarly defined for $F^{\prime}$ and $G^{\prime}$. We note that $P \cong T_{1}^{\prime} / \alpha\left(T_{1}\right)$ and $Q \cong T_{2}^{\prime} / \beta\left(T_{2}\right)$. Let $\eta \in \operatorname{Hom}_{A|\mathcal{G}|}(P, Q)$ and $\Gamma(\eta)$ be the superlattice of $\alpha\left(T_{1}\right) \times \beta\left(T_{2}\right)$ in $T_{1}^{\prime} \times T_{2}^{\prime}$ such that $\Gamma(\eta) / \alpha\left(T_{1}\right) \times \beta\left(T_{2}\right)$ is the graph of $\eta$. We have the following commutative diagram with exact rows:

$$
\begin{aligned}
& \begin{array}{rlccccc}
0 \rightarrow T_{2} & \stackrel{i}{\rightarrow} & T_{1} \times T_{2} & \stackrel{j}{\rightarrow} & T_{1} & \rightarrow & 0 \\
\| & & \downarrow \varepsilon & & \downarrow \alpha \\
0 \rightarrow T_{2} & \rightarrow & \Gamma(\eta) & \rightarrow & T_{1}^{\prime} & \rightarrow & 0 \\
& \downarrow \beta
\end{array} \\
& 0 \rightarrow T_{2}^{\prime} \stackrel{i^{\prime}}{\rightarrow} T_{1}^{\prime} \times T_{2}^{\prime} \stackrel{j^{\prime}}{\rightarrow} T_{1}^{\prime} \quad \rightarrow \quad 0
\end{aligned}
$$

where $i, i^{\prime}$ are the canonical injections, $j, j^{\prime}$ the canonical projections and $\varepsilon$ the composite map $T_{1} \times T_{2} \stackrel{\alpha \times \beta}{\rightarrow} \alpha\left(T_{1}\right) \times \beta\left(T_{2}\right) \hookrightarrow \Gamma$. Then the functor $M L$ gives the 
following commutative diagram, whose rows are exact by Lemma 1:

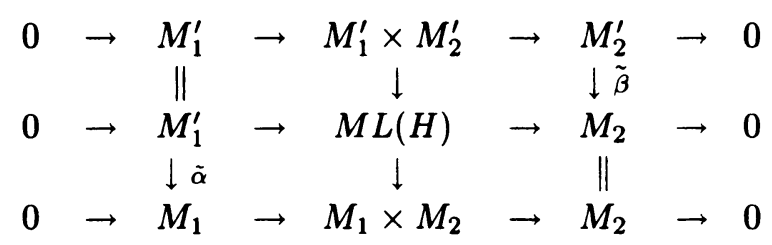

where $H \in F^{A}(B)$ is such that $T(H) \cong \Gamma(\eta)$ (cf. [10]). By the above diagram we have a morphism $M L(Q)=M_{2} / \tilde{\beta} M_{2}^{\prime} \rightarrow M L(P)=M_{1} / \tilde{\alpha} M_{1}^{\prime}$, which does not depend on the choice of $H$; we denote it by $\theta(\eta)$. By construction we see easily that $\theta: \operatorname{Hom}_{A[\mathfrak{E}]}(P, Q) \rightarrow \operatorname{Hom}_{E}(M L(Q), M L(P))$ is a bijection. Let $\eta_{1}, \eta_{2} \in$ $\operatorname{Hom}_{A[\mathcal{E}]}(P, Q)$. As the exact sequence $0 \rightarrow T_{2} \rightarrow \Gamma\left(\eta_{1}+\eta_{2}\right) \rightarrow T_{1}^{\prime} \rightarrow 0$ is the Baer sum of the extensions $0 \rightarrow T_{2} \rightarrow \Gamma\left(\eta_{i}\right)-T_{1}^{\prime} \rightarrow 0(i=1,2)$, we see that $\theta$ is a homomorphism using the functor $M L$. Clearly $\theta$ is an $A$-isomorphism.

2. One-dimensional formal $A$-modules. Let $v$ be the valuation of $\bar{L}$ which is normalized so that $v(\pi)=1$. Here we assume that all formal $A$-modules are one-dimensional. Let $u=\pi+\sum_{\nu=1}^{\infty} b_{\nu} T^{\nu}$ be a special element in $E$. We write $\left(u^{-1} \pi\right)^{*}(x)$ for the element $f(x)$ of $L[[x]]$ such that $f(0)=0$ and $\pi x=\pi f(x)+$ $\sum_{\nu=1}^{\infty} b_{\nu} f^{\sigma^{\nu}}\left(x^{q^{\nu}}\right)$. Then $F(x, y)=f^{-1}(f(x)+f(y))$ is a formal $A$-module over $B$. This shows that the strong isomorphism classes of formal $A$-modules over $B$, of $A$ height $h$, correspond bijectively to the special elements of the form $\pi+\sum_{\nu=1}^{h} b_{\nu} T^{\nu}$, where $b_{1}, \ldots, b_{h-1} \in \mathfrak{p}$ but $b_{h}$ is a unit of $B$ (cf. Cox [1]). Let $F$ be a formal $A$-module of $A$-height $h$ defined over $B$. We write $\Lambda_{F, m}=\operatorname{Ker}\left[\pi^{m}\right]_{F}=\{x \in$ $\left.\overline{\mathfrak{p}} \mid\left[\pi^{m}\right]_{F}(x)=0\right\}$ for $m \geq 0$, which is a finite subgroup of order $q^{h m}$ in $F(\overline{\mathfrak{p}})$.

THEOREM 2. Let $u_{1}=\pi+\sum_{i=1}^{h} b_{i} T^{i}$ and $u_{2}=\pi+\sum_{i=1}^{h} c_{i} T^{i}$ be special elements of $E$ such that $b_{i}, c_{i} \in \mathfrak{p}(1 \leq i \leq h-1)$ and $b_{h}, c_{h}$ are units of $B$. Let $f_{1}(x)=\left(u_{1}^{-1} \pi\right)^{*}(x)=\sum_{n=0}^{\infty} a_{n} x^{q^{n}}, f_{2}(x)=\left(u_{2}^{-1} \pi\right)^{*}(x)=\sum_{n=0}^{\infty} a_{n}^{\prime} x^{q^{n}}$ and $\psi=f_{2}^{-1} \circ f_{1}$. Let $m$ be an integer such that $u_{1} \equiv u_{2} \bmod \mathfrak{p}^{m}$ but $u_{1} \not \equiv u_{2} \bmod \mathfrak{p}^{m+1}$. Put $w_{i}=\left(b_{i}-c_{i}\right) / \pi^{m}$ for $1 \leq i \leq h$ and let $e(1 \leq e \leq h)$ be such that $w_{i} \in \mathfrak{p}$ for $1 \leq i \leq e-1$ and $w_{e}$ is a unit. Then the convergence domain of $\psi$ contains $\left\{x \in \overline{\mathfrak{p}} \mid v(x)>q^{-e} r^{-m+1}(r-1)^{-1}\right\}$, where $r=q^{h}$.

For the proof of Theorem 2 we need the following

LEMMA 2. Assume the same hypothesis as in Theorem 2 , and put $A_{n}=a_{n}-a_{n}^{\prime}$. Then we have $v\left(A_{n}\right) \geq(m-1)-[(n-e) / h]$ for $n \geq 0$, where $[\alpha]$ denotes the largest integer not exceeding $\alpha$.

ProOF. We proceed by induction on $n$. First we note that $v\left(a_{t}^{\prime}\right) \geq-[t / h]$ by [1, Proposition 4.1.1]. By the definition of $f_{1}$ and $f_{2}$ we can show that

$$
A_{n}=-\sum_{i=1}^{h} \pi^{-1} b_{i} A_{n-i}^{\sigma^{i}}-\pi^{m-1} \sum_{i=1}^{h} w_{i} a_{n-i}^{\sigma^{2}} .
$$

Then it is clear that $v\left(A_{n}\right) \geq m$ for $0 \leq n \leq e$. Hence we may assume that the assertion of our lemma holds for $n^{\prime}$ with $n^{\prime}<\bar{n}=h(j-1)+e+k$, where $0 \leq k<h$ 
and $j \geq 1$. We have $v\left(\pi^{-1} b_{i} A_{n-i}\right) \geq m-1-[(n-i-e) / h] \geq m-j$ for $1 \leq i \leq h-1$ and $v\left(\pi^{-1} b_{h} A_{n-h}\right) \geq m-j$. Noting $e+k<2 h$, we have

$$
v\left(\pi^{m-1} w_{i} a_{n-i}^{\prime \sigma^{2}}\right) \geq m-1-[(n-h) / h]=m-j-[(e+k-h) / h] \geq m-j .
$$

Therefore $v\left(A_{n}\right) \geq m-j=(m-1)-[(n-e) / h]$. This completes our proof by induction.

PROOF OF THEOREM 2. We can write $\psi(x)=\sum_{n=0}^{\infty} \alpha_{n} x^{n(q-1)+1}$ with $\alpha_{n} \in L$ by Lubin [7, p. 475]. Let $\xi$ be an element of $\bar{p}$ such that $\xi^{q^{e} r^{m-1}(r-1)}=\pi$. Put $\beta_{n}=\alpha_{n} \xi^{n(q-1)+1}$. By induction on $n$ we shall show that $v\left(\beta_{n}\right) \geq 1 /(r-1)$. Let $R$ be the set whose points are sequences $\mathfrak{n}=\left(n_{0}, n_{1}, n_{2}, \ldots\right)$, where $n_{i}$ are nonnegative integers for all $i$ and $n_{i}=0$ for almost all $i$. For $\mathfrak{n} \in R$, define $|\mathfrak{n}|=\sum_{k=0}^{\infty} n_{k}$, $\mathrm{n}^{*}=\sum_{k=0}^{\infty} k n_{k}$ and $C(\mathfrak{n})=|\mathfrak{n}| ! /\left(\prod_{k=0}^{\infty}\left(n_{k} !\right)\right)$. They are rational integers. We define an element $\alpha^{n}$ of $L$ to be $\prod_{k=0}^{\infty} \alpha_{k}^{n_{k}}$. Put $Q_{s}=\left(q^{s}-1\right) /(q-1)$. Let $t$ be an integer such that $Q_{t}<N+1 \leq Q_{t+1}$. On comparing the coefficients of $x^{(N+1)(q-1)+1}$, we get by the equation $f_{2}(\psi(x))=f_{1}(x)$ that

$$
\alpha_{N+1}+\sum_{k=1}^{t} a_{k}^{\prime}\left(\sum_{k} C(\mathfrak{n}) \alpha^{\mathfrak{n}}\right)= \begin{cases}0 & \text { if } N+1<Q_{t+1} \\ A_{t+1} & \text { if } N+1=Q_{t+1}\end{cases}
$$

where the sum $\sum_{k}$ is taken over all $\mathfrak{n} \in R$ such that $|\mathfrak{n}|=q^{k}$ and $\mathfrak{n}^{*}=N+1-Q_{k}$. We have easily by Lemma 2 that $v\left(A_{1}\right) \geq m-1$ if $e=1$ and $v\left(A_{1}\right) \geq m$ if $e>1$. Then

$$
v\left(\beta_{1}\right)=v\left(\xi^{q} \alpha_{1}\right) \geq q^{1-e} r^{1-m}(r-1)^{-1}+v\left(A_{1}\right) \geq 1 /(r-1) .
$$

Therefore by induction hypothesis we assume that $v\left(\beta_{n}\right) \geq 1 /(r-1)$ for $1 \leq n \leq N$. For $\mathfrak{n}=\left(n_{0}, n_{1}, n_{2}, \ldots\right) \in R$ with $|\mathfrak{n}|=q^{k}(k \geq 1)$ and $\mathfrak{n}^{*}=N+1-Q_{k}$, let

$$
D_{k, \mathfrak{n}}^{N+1}=\xi^{(N+1)(q-1)+1} a_{k}^{\prime} C(\mathfrak{n}) \alpha^{n}=a_{k}^{\prime} C(\mathfrak{n}) \xi^{n_{0}} \prod_{k=1}^{\infty} \beta_{k}^{n_{k}} .
$$

Now let $g(x)=r^{x}(r-1)^{-1}-x ;$ it is clear that $g(n) \geq 1 /(r-1)$ for all integers $n$ and $g(n)=1 /(r-1)$ if and only if $n=0$ or $n=1$. Now if $n_{0}=0$, then

$$
v\left(D_{k, \mathbf{n}}^{N+1}\right) \geq v\left(a_{k}^{\prime}\right)+v(C(\mathrm{n}))+q^{k} /(r-1) \geq g([k / h]) \geq 1 /(r-1) .
$$

If $n_{0} \neq 0$, then $0<n_{0}<q^{k}$. Writing $q=p^{j}, n_{0}=q^{s} d$ with $q \not d$ and $d=\dot{p}^{j^{\prime}} d_{1}$ with $\left(p, d_{1}\right)=1$, we easily get

$$
\operatorname{ord}_{p}\left({ }_{q^{k}} C_{n_{0}}\right)=j(k-s)-j^{\prime} \geq k-s .
$$

Clearly ${ }_{q^{k}} C_{n_{0}}$ is a divisor of $C(\mathbf{n})$ and $q^{s} \leq q^{k}-n_{0}$. Therefore

$$
\begin{aligned}
v\left(D_{k, \mathrm{n}}^{N+1}\right) & \geq v\left(a_{k}^{\prime}\right)+v(C(\mathrm{n}))+n q^{-e} r^{1-m}(r-1)^{-1}+\left(q^{k}-n_{0}\right)(r-1)^{-1} \\
& >-[k / h]+(k-s)+q^{s}(r-1)^{-1} \geq g([s / h]) \geq(r-1)^{-1} .
\end{aligned}
$$

Let us now assume $N+1=Q_{t+1}$. Then, by Lemma 2, we have

$$
v\left(\xi^{(N+1)(q-1)+1} A_{t+1}\right) \geq g(-(m-1)+[(t+1-e) / h]) \geq(r-1)^{-1} .
$$

In view of $\left({ }^{*}\right)$, we have thus established that $v\left(\beta_{N+1}\right) \geq 1 /(r-1)$; therefore $v\left(\beta_{n}\right) \geq$ $1 /(r-1)$ for all $n \geq 1$. As $\psi(x)=\sum_{n=0}^{\infty} \beta_{n}(x / \xi)^{n(q-1)+1}$, the proof is completed.

REMARK. By further computations we can show that $v\left(\beta_{Q_{h o+e}}\right)=1 /(r-1)$ for $s \geq m$. Therefore the convergence domain of $\psi$ is $\left\{x \in \overline{\mathfrak{p}} \mid v(x)>q^{-e} r^{1-m}(r-1)^{-1}\right\}$. 
COROLlaRY. Assumptions and notation being as in Theorem 2 , let $F_{i}(x, y)=$ $f_{i}^{-1}\left(f_{i}(x)+f_{i}(y)\right)(i=1,2)$. Then $\psi$ defines an $A[\mathfrak{G}]$-isomorphism $\Lambda_{F_{1}, m} \rightarrow \Lambda_{F_{2}, m}$.

As $v(x) \geq r^{1-m}(r-1)^{-1}$ for $x \in A_{F_{1}, m}$, this is clear.

THEOREM 3. Let $F$ and $G$ be one-dimensional formal $A$-modules of the same $A$-height $h$ defined over $B$ and $f, g$ be the logarithms of $F, G$, respectively. Then every element of $\operatorname{Hom}_{A|\mathcal{G}|}\left(\Lambda_{F, m}, \Lambda_{G, m}\right)$ is of the form $g^{-1} \circ c f$ for some $c \in B$. If $f$ and $g$ are of type $u_{1}=\pi+\sum_{i=1}^{h} b_{i} T^{i}$ and $u_{2}=\pi+\sum_{i=1}^{h} c_{i} T^{i}$, respectively (cf. $[\mathbf{1}$, p. 295]), then $g^{-1} \circ c f \in \operatorname{Hom}_{A[\mathcal{G}]}\left(\Lambda_{F, m}, \Lambda_{G, m}\right)$ for $c \in B$ if and only if $u_{2} c \equiv c u_{1}$ $\bmod \mathfrak{p}^{m}$.

PROOF. As $M(F) \cong E / E u_{1}$ and $M\left(\Lambda_{F, m}\right) \cong E /\left(E u_{1}+E \pi^{m}\right)$, we get easily by Theorem 1 that

$$
\operatorname{Hom}_{A[\mathfrak{G}]}\left(\Lambda_{F, m}, \Lambda_{G, m}\right) \cong\left\{c \in B \mid u_{2} c \equiv c u_{1} \bmod \mathfrak{p}^{m}\right\} / \mathfrak{p}^{m} .
$$

Let $c \in B$ be such that $u_{2} c \equiv c u_{1} \bmod p^{m}$. We assume $v(c)=s \leq m$ and write $c=b \pi^{s}$ with a unit $b$ in $B$. Let $u^{\prime}=b u_{1} b^{-1}$. Then $u^{\prime}$ is special and $u^{\prime} \equiv u_{2}$ $\bmod \mathfrak{p}^{m-s}$. Let $f_{1}(x)=\left(u^{\prime-1} \pi\right)^{*}(x)$ and $F_{1}(x, y)=f_{1}^{-1}\left(f_{1}(x)+f_{1}(y)\right)$. Then $g^{-1} \circ c f=\left(g^{-1} \circ f_{1}\right)\left[\pi^{s}\right]_{F_{1}} \circ\left(f_{1}^{-1} \circ b f\right)$, where $f_{1}^{-1} \circ b f: F \rightarrow F_{1}$ is an isomorphism. By the Corollary above, $g^{-1} \circ c f$ defines an element $\eta(c)$ of $\operatorname{Hom}_{A[\mathcal{G}\}}\left(\Lambda_{F, m}, \Lambda_{G, m}\right)$ : clearly $\eta(c)=\eta\left(c^{\prime}\right)$ if and only if $c \equiv c^{\prime} \bmod \mathfrak{p}^{m}$. Our assertion is now obvious.

REMARK. For a formal $A$-module $F$ over $B$ of finite $A$-height $h$, we have the results which are completely analogous to those in Fontaine $[3]$. Let $\rho: \mathfrak{G} \rightarrow$ $\operatorname{Aut}_{A}(T(F))\left(\cong \mathrm{GL}_{h}(A)\right)$ be the $\pi$-adic representation attached to $F$. Then by [3] we have

(1) $\rho(\mathfrak{G}) \supset A^{\times}$. Therefore $\mathfrak{G}$-endomorphisms of $\Lambda_{F, m}$ are $A[\mathfrak{G}]$-endomorphisms.

(2) For $h=1$ or 2, applying our Theorem 3 we can determine the closed subgroup $\rho(\mathfrak{G})$ of $\mathrm{GL}_{h}(A)$ (up to an isomorphism) by the special element of $F$.

\section{REFERENCES}

1. L. H. Cox, Formal A-modules over p-adic integer rings, Compositio Math. 29 (1974), 287-308.

2. J.-M. Decauwert, Classification des A-modules formels, C. R. Acad. Sci. Paris 282 (1976), 1413 1416.

3. J.-M. Fontaine, Points d'ondre fini d'un groupe formel sur une extension non ramifiée de $Z_{p}$, Bull. Soc. Math. France 37 (1974), 75-79.

4. __, Groupes finis commutatifs sur les vectuers de Witt, C. R. Acad. Sci. Paris 280 (1975). 1423-1425.

5. __ Groupes p-divisibles sur les corps locaux, Astérisque 47-48, Soc. Math. de France, 1977.

6. T. Honda, On the theory of commutative formal groups, J. Math. Soc. Japan 22 (1970), 213246.

7. J. Lubin, One parameter formal Lie groups over p-adic integer rings, Ann. of Math. (2) 80 (1964), 464-484.

8. _ Galois endomorphisms of the torsion subgroup of certain formal groups, Proc. Amer. Math. Soc. 20 (1969), 229-331.

9. F. Oort, Dieudonné modules of finite local group schemes, Nederl. Akad. Wetensch. Proc. Ser. A 77 (1974), 284-292.

10. W. Waterhouse, On p-divisible groups over complete valuation rings, Ann. of Math. (2) 95 (1972), 55-65.

Department of Mathematics, College of General Education, Tôhoku UniVERSITY, KAWAUCHI, SENDAI 980, JAPAN 